\title{
Association of Multiple DRD2 Polymorphisms with Anorexia Nervosa
}

\begin{abstract}
Andrew W Bergen*,1,16 Meredith Yeager², Robert A Welch², Kashif Haque', J Kelly Ganjei ${ }^{3}$, Marianne BM van den Bree ${ }^{4}$, Chiara Mazzanti ${ }^{5}$, Irma Nardi ${ }^{6}$, Manfred M Fichter ${ }^{7}$, Katherine A Halmi ${ }^{8}$, Allan S Kaplan', Michael Strober ${ }^{10}$, Janet Treasure "', D Blake Woodside', Cynthia M Bulik ${ }^{12}$, Silviu-Alin Bacanu ${ }^{13}$, Bernie Devlin ${ }^{13}$, Wade H Berrettini ${ }^{14}$, David Goldman ${ }^{15}$ and Walter H Kaye ${ }^{13}$

'Division of Cancer Epidemiology and Genetics, National Cancer Institute, Bethesda, MD, USA (current affiliation); ${ }^{2}$ Core Genotyping Facility, Advanced Technology Center, National Cancer Institute, Gaithersburg, MD, USA; ${ }^{3}$ TissueGene, Inc., Gaithersburg, MD, USA; ${ }^{4}$ Department of Psychological Medicine, Cardiff University, Cardiff, UK; ${ }^{5}$ Laboratory of Human Cytogenetics, Cancer Research UK, London, UK; ${ }^{6}$ Laboratorio di Biologia Cellulare e dello Sviluppo, University of Pisa, La Fontina Ghezzano, Pisa, Italy; ${ }^{7}$ Klinik Roseneck, Hospital for Behavioral Medicine, Affiliated with University of Munich, Prien, Germany; ${ }^{8}$ New York Presbyterian Hospital-Westchester, Weill Medical College of Cornell University, White Plains, NY, USA; ' Department of Psychiatry, University Health Network, Toronto General Hospital, Toronto, ON, Canada;

${ }^{10}$ Neuropsychiatric Institute and Hospital, School of Medicine, University of California at Los Angeles, Los Angeles, CA, USA; ${ }^{11}$ Institute of Psychiatry, Kings College, London, England, UK; ${ }^{12}$ Department of Psychiatry, University of North Carolina, Chapel Hill, NC, USA; ${ }^{13}$ Department of Psychiatry, University of Pittsburgh School of Medicine, Pittsburgh, PA, USA; ${ }^{14}$ Center for Neurobiology and Behavior, Department of Psychiatry, University of Pennsylvania Health System, Clinical Research Building, Philadelphia, PA, USA; ${ }^{15}$ Laboratory of Neurogenetics, National Institute of Alcohol Abuse and Alcoholism, Rockville, MD, USA
\end{abstract}

\begin{abstract}
To investigate whether the dopaminergic system plays a role in the etiology of anorexia nervosa (AN) via the dopamine D2 receptor, we investigated association and transmission disequilibrium at seven single-nucleotide polymorphisms (SNPs) spanning about $75 \mathrm{kbp}$ of the gene DRD2. We studied 19I probands with a DSM-IV diagnosis of AN, 457 parents and affected relatives with a DSM-IV eating disorder diagnosis, and 98 unrelated, female, normal weight controls. The $-|4| \mathrm{C} /-$ insertion/deletion $(-|4|$ Indel), previously shown to affect DRD2 transcription efficiency, and multiple exon seven polymorphisms, one of which has previously been shown to affect DRD2 transcript stability, exhibited statistically significant association with diagnosis in haplotype transmission disequilibrium and in haplotype case: control analyses. Significant linkage disequilibrium between the - I4I Indel and two exon seven SNPS (939Y and 957Y) was observed over a distance of $>50 \mathrm{kbp}$ in the AN probands but not in the controls. Genetically transmitted variation in D2 dopamine receptor expression mediated by functional polymorphisms affecting transcription and translation efficiency may play a role in vulnerability to AN.

Neuropsychopharmacology (2005) 30, 1703-1710. doi: I0.1038/s..npp. I3007 I9; published online 25 May 2005
\end{abstract}

Keywords: anorexia nervosa; case-control studies; female; haplotypes; linkage disequilibrium; dopamine D2

\section{INTRODUCTION}

Anorexia nervosa (AN), a disorder with typically an adolescent age of onset, is characterized by pathologic eating behavior, by the relentless pursuit of thinness leading to emaciation, and by a mortality rate substantially greater than other psychiatric disorders (Sullivan, 1995). AN

\footnotetext{
*Correspondence: Dr AW Bergen, Division of Cancer Epidemiology and Genetics, National Cancer Institute, 6120 Executive Boulevard, Bethesda, MD 20892-7236, USA, Tel: + | 30 | 435 76|4, Fax: + | 30 | 402 4489, E-mail: bergena@mail.nih.gov

${ }^{16}$ This research originated in part through the Price Foundation Collaboration and does not represent the opinion of the $\mathrm{NIH}$, the DHHS or the Federal Government.

Received 18 August 2003; revised 31 January 2005; accepted 7 February 2005

Online publication: 15 February 2005 at http://www.acnp.org/citations/ Npp02 I505030377/default.pdf
}

patients often display characteristic temperament and personality traits that include obsessionality, perfectionism, and anxiety (Halmi et al, 2000; Bulik et al, 2003; Kaye et al, 2004). AN is familial, with a relative risk of $\sim 11$ and an additive genetic component of $\sim 60 \%$ (Strober et al, 2000; Lilenfeld et al, 1998; Wade et al, 2000; Klump et al, 2001; Kortegaard et al, 2001).

Based on this evidence for genetic susceptibility, a multicenter collaborative group, the Price Foundation Collaborative Group (PFCG), has collected a large sample of eating disorder families $(N=196)$ ascertained from AN probands, known as the AN affected relative pair (AN-ARP) data set (Kaye et al, 2000). Using these families, the PFCG has reported suggestive linkage to chromosome 1p34-36 for restricting AN (Grice et al, 2002), as well as to 1q, 2q, and 13p (Devlin et al, 2002). In addition to linkage studies, the PFCG has investigated candidate susceptibility genes, such as those influencing monoamine and neuropeptide function (Bergen 
et al, 2003b). For example, we have analyzed HTR1D, OPRD1, and HCRTR1, all biological candidate genes found in the chr1p34-36 linkage region (Bergen et al, 2003a), and have identified significant case : control and TDT association to AN diagnosis at HTR1D and OPRD1 (Bergen et al, 2003a).

Disturbances of CNS dopamine metabolism could contribute to symptoms in AN. Dopaminergic neuronal function modulates feeding behavior (Szczypka et al, 1999, 2000), motor activity (Kalivas, 1993), and rewardmotivated (Blum et al, 1995; Salamone, 1996) and drugseeking behavior (Bardo et al, 1996). Aside from their wellknow disturbances of feeding and weight maintenance, individuals with AN have stereotypic and hyperactive motor behavior (Kron et al, 1978; Beumont et al, 1994), anhedonic, obsessional personalities, and reduced novelty seeking (Sohlberg and Strober, 1994). Moreover, we have shown that individuals who have recovered from AN exhibit significantly lower CSF homovanillic acid concentrations than individuals with a history of $\mathrm{BN}$ diagnosis or control individuals (Kaye et al, 1999).

It is plausible that D2 receptors, which are most prominent in the striatum, nucleus accumbens, and olfactory tubercle (Mukherjee et al, 1996), could play a role in AN. In addition to their potential effects on feeding behavior, the striatal dopamine system affects hyperactivity, and dopamine neurons in the nucleus accumbens/limbic regions are involved in reward alterations and novelty seeking (Wise and Bozarth, 1984; Comings and Blum, 2000). The dopamine receptor D2 is a seven transmembrane $G$ protein linked receptor that binds dopamine and inhibits adenylate cyclase (Kebabian and Calne, 1979). The literature suggests this receptor acts as an autoreceptor on dopaminergic cell bodies and as a postsynaptic receptor on dopaminergic targets (Khan et al, 1998; Usiello et al, 2000). Extending over $65 \mathrm{~kb}$ on chromosome $11 q 23$, with an intron of approximately $50 \mathrm{~kb}$ separating exons one and two, DRD2 has been investigated as a candidate biological susceptibility locus in linkage and association studies in many psychiatric diseases (Cravchik and Goldman, 2000). Functional polymorphisms at DRD2 include the -141 Indel polymorphism, shown to affect DRD2 transcription efficiency (Arinami et al, 1997), the exon 7 nonsynonymous nucleotide polymorphism (SNP), 932C $>$ G (Itokawa et al, 1993), shown to impair dopamine-mediated inhibition of forskolin-induced adenylate cyclase activity (Cravchik et al, 1996) and the exon 7 synonymous SNP, 957C > T (Cargill et al, 1999), shown to affect DRD2 transcript stability and translational efficiency (Duan et al, 2003). As DRD2 is a plausible candidate gene for AN, we genotyped seven SNPs that physically span the gene in both case: control and family samples and evaluated association to AN phenotype.

\section{MATERIALS AND METHODS}

\section{Samples}

We recruited 196 probands, as well as relatives affected with eating disorders and parents, through the use of clinical services, patient databases, and advertisements (Kaye et al, 2000). Ascertainment criteria required at least 3 years duration of DSM-IV AN diagnosis excluding the amenorrhea criterion, age between 13 and 65 years (Kaye et al, 2000). Diagnoses were obtained using the Structured
Interview for Anorexic and Bulimic Disorders (Fichter et al, 1998). Exclusion criteria included onset of AN after age 25 or a lifetime diagnosis of a number of neurological, psychiatric, or metabolic disorders that could affect cognition, appetite, eating behavior, and body weight, for example, diabetes mellitus. Binge eating is commonly observed in individuals with a diagnosis of $\mathrm{AN}$, and constitutes an exclusion criterion, because it would increase heterogeneity among the proband population (Kaye et al, 2000). Most probands were female (95\%), diagnosed with either DSM-IV Restricting AN (RAN) or Purging AN (PAN) subtypes (55 and 45\%, respectively). Affected relatives could fulfill the same ascertainment and exclusionary criteria as probands (86 affected relatives), or could fulfill a broadened diagnosis of AN with binge eating, DSM-IV bulimia nervosa or subthreshold eating disorder (59, 47, and 37 affected relatives, respectively) (Kaye et al, 2000). Families originated from North America (70\%), the United Kingdom (11\%), and Germany (19\%). Unrelated European-American females ('EAF') were recruited through advertisements and used for a control sample in case:control comparisons. Control ascertainment excluded individuals over $120 \%$ ideal weight or who met lifetime criteria for Axis I disorders as assessed by the Structured Clinical Interview for DSM-III-R (First et al, 1996). Informed written consent was obtained from all study participants and all sites received approval from their local institutional review board as previously described (Kaye et al, 2000). The EAF control sample was interviewed and provided a blood sample as part of an IRB approved protocol that included informed written consent. DNA was available from a total of 191 probands, 457 parents and affected relatives, and 98 controls. For single and multilocus DRD2 TDT analysis, there were 253 nuclear families available. Unrelated Centre Étude Polymorphism Humaine (CEPH) and Caucasian Variation (CV) Panel DNA samples obtained from the Coriell Cell Repositories (http://locus.umdnj.edu/) were used for resequencing, genotype assay development and as individual control DNAs for genotyping assays.

\section{Selection of DRD2 Polymorphisms}

Sequence variation at the DRD2 locus was identified using public literature, sequence, and variation databases. To verify published SNPs around exon seven, 32 unrelated CEPH DNA samples were sequenced from -187 bases before its start to 350 bases into exon seven. Seven DNA polymorphisms at the DRD2 locus were selected for genotyping (Figure 1): (1) the $-141 \rightarrow \mathrm{C}$ or Indel, NCBI SNP ID: rs1799732 (Arinami et al, 1997); (2) IVS22730T > C, NCBI SNP ID: rs1800498 (Kidd et al, 1998); (3) 932C $>$ G, exon 7, S311C, NCBI SNP ID: rs1801028 (Itokawa et al, 1993); (4) 939C $>\mathrm{T}$, exon 7, H313, NCBI SNP ID: rs6275 (Sarkar et al, 1991); (5) 957C > T, exon 7, P319, NCBI SNP ID: rs6277 (Cargill et al, 1999); (6) 725 bp 3' of STP G > T or Ex8-408 3'UTR, NCBI SNP ID: rs6278 (Cargill et al, 1999); and (7) 10620C $>$ T $3^{\prime}$ of STP C $>$ T or TaqIA, E713K in ANKK1, NCBI SNP ID: rs1800497 (Grandy et al, 1989). All positions, with the exception of the TaqIA polymorphism, are relative to the A of the ATG initiation codon, long isoform cDNA with substitution type indicated when appropriate (NT_033899). All SNPs except for one $(932 \mathrm{C}>\mathrm{G})$ were genotyped in the case:control samples 


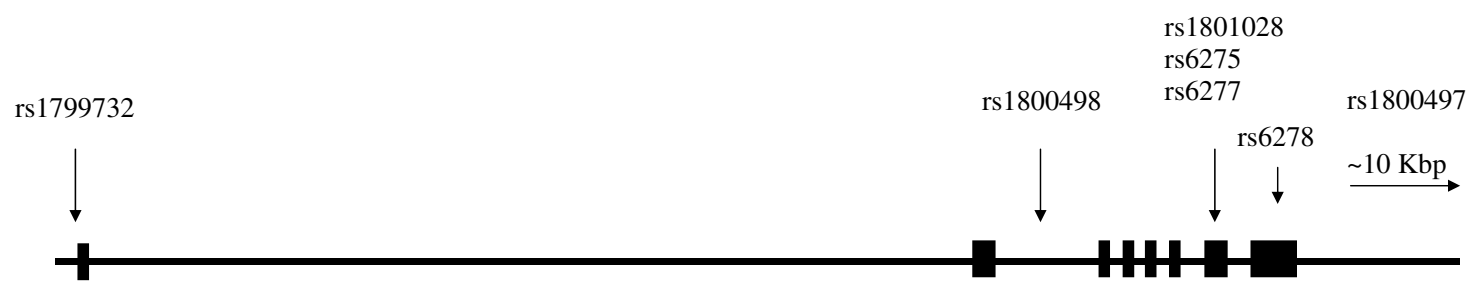

Figure I DRD2 exons I-8 and genotyped DRD2 SNPs.

(Supplementary Table 1), while four SNPs were also genotyped in families of AN probands (-141 Indel, 932C $>$ G, 939C $>\mathrm{T}$ and $957 \mathrm{C}>\mathrm{T}$ ).

\section{Genotyping}

Of the AN proband samples, $N=183,132,183,191,191,132$, and 132 DNA samples were subjected to genotyping at the seven DRD2 SNPs, respectively. DNA samples from $N=457$ AN-ARP family members were genotyped at the -141 Indel, $932 \mathrm{C}>\mathrm{G}, 939 \mathrm{C}>\mathrm{T}$ and $957 \mathrm{C}>\mathrm{T}$ SNPs. Of the EAF samples, $N=98$ DNA samples were genotyped at six DRD2 SNPs, excluding the 932C $>$ G SNP. A duplicate sample of 67 DNAs was drawn from these groups and genotyped to assess the reproducibility of SNPs genotyped in the case:control sample. SNPs genotyped in the family sample were evaluated for apparent non-Mendelian transmissions.

SNPs were genotyped using the $5^{\prime}$ exonuclease assay $\left(\operatorname{TaqMan}^{\mathrm{TM}}\right.$ ) (Morin et al, 1999), with the exception of the 932C > G SNP, which was genotyped as previously described (Fujiwara et al, 1997). 5' exonuclease probes and primers were chosen using ProbeITY (Celadon Laboratories, College Park, MD) and were synthesized by Applied Biosystems (Foster City, CA). Quality control procedures for $5^{\prime}$ exonuclease genotyping included no template controls, sequenced genotype controls, and duplicated DNA samples in different plates for genotype assay quality control. Primer and probe sets for SNPs were as follows: the $-141 \rightarrow \mathrm{C}$ or Indel: forward primer- $5^{\prime}$-CAAAACAAGGGATGGCGGAA TC- $3^{\prime}$, reverse primer - 5' -CCACCAAAGGAGCTGTACCT-3', probe 1-VIC-CTACCCGTTCCAGGCCG-Tamra, probe 2-FAM-CTACCCGTTCAGGCCG-TAMRA; IVS2-2730T > C: forward primer - $5^{\prime}$-AGCAGAGGAAGGAGTG-3', reverse primer - $5^{\prime}$-AATGATGCCTGGATGC-3', probe 1 -FAM-tcc ctagtcAaacccaaggct-TAMRA, probe 2 - TET-tccctagtcGaacc caaggc-TAMRA; 939C $>\mathrm{T}$ : forward primer-5'-CTGAC TCTCCCCGAC-3', reverse primer - $5^{\prime}$-CTTGGGGTGGTCTT TG-3', probe 1-FAM-ccaccaCggtctccacggc-TAMRA, probe 2 -VIC-ccaccaTggtctccacggc-TAMRA; 957C $>\mathrm{T}$ : forward primer - $5^{\prime}$-CCCATTCTTCTCTGGTTT-3', reverse primer$5^{\prime}$-CTGACTCTCCCCGAC-3', probe 1 -FAM-cggggctgtcAg gagtgc-TAMRA, probe 2-VIC-cggggctgtcGggagt-TAMRA; $725 \mathrm{bp} 3^{\prime}$ of STP G>T: forward primer - $5^{\prime}$-TATGGGGA GAGGAACTC-3', reverse primer $-5^{\prime}$-GAGAAGGGATACA TTGCA-3', probe 1-FAM-agcccacctGctgcc-TAMRA, probe 2-TET-agcccaccctTctgcctt-TAMRA; TaqIA: forward primer - $5^{\prime}$-CAACACAGCCATCCTC- $3^{\prime}$, reverse primer $-5^{\prime}$ TCACTCCATCCTGGAC-3', probe 1 -FAM-ctggtcAagg
caggctc-TAMRA, probe 2-VIC-tggtcGaggcaggcgc-TAMRA (Figure 1). General conditions per reaction for PCR and end point-read $\mathrm{TaqMan}^{\mathrm{TM}}$ were as previously described (Morin et al, 1999), with the exception of IVS2-2730T $>\mathrm{C}$ and +725 $3^{\prime}$ SNPs; PCR reaction conditions for these SNPs were optimized with an annealing temperature of $55^{\circ} \mathrm{C}$. Genotype determination was performed on an Applied Biosystems Sequence Detector 7700 (Applied Biosystems, Foster City, CA).

\section{Statistical Procedures}

Tests of Hardy-Weinberg equilibrium (HWE) in the AN, RAN, PAN, and EAF samples were performed using Pearson's $\chi^{2}$ test. Pearson's $\chi^{2}$ tests were also used to test for independence of diagnostic status and either genotype or allele counts using SISA (http://home.clara.net/sisa/). Pairwise haplotypes at the -141 Indel, IVS2-2730T $>C$, 939C $>\mathrm{T}, 957 \mathrm{C}>\mathrm{T},+7253^{\prime} \mathrm{G}>\mathrm{T}$ and TaqIA $\mathrm{C}>\mathrm{T}$ DRD2 SNPs from AN proband (mean $N=127$ ), RAN probands (mean $N=66$ ), PAN probands (mean $N=61$ ) and EAF (mean $N=80$ ) samples were estimated using an Expectation-Maximization algorithm (Excoffier and Slatkin, 1995) and pairwise linkage disequilibrium and the significance of haplotype frequency differences were estimated using Pearson's $\chi^{2}$ test and, by permutation, using the MCMC algorithm (Raymond and Rousset, 1995), implemented in Arlequin (Schneider et al, 2000). S.A.G.E. and Pedcheck were used to identify apparent non-Mendelian transmissions in the AN-ARP family sample (Elston, 2001; O'Connell and Weeks, 1998).

The FBAT program was used for single and multilocus SNP TDT analysis in the family data set (Lange and Laird, 2002a, b). Both single SNP and multilocus haplotype-based TDT test used all individuals of the AN-ARP data set that had been genotyped, with probands and those affected relatives who had a diagnosis of DSM-IV AN, either RAN or PAN defined as affected individuals. $P$-values were obtained asymptotically for single locus tests, and asymptotically and by permutation for haplotype tests. To calculate power for case: control and TDT tests, we assumed the polymorphisms directly affect liability to disease, a prevalence of $1 \%$ and the estimated frequency of each allele (p) was the true frequency in the study population. Power was computed under four disease inheritance models: recessive, dominant, additive, and multiplicative. To evaluate the power to test for violations of independence between alleles and disease status by using the sample of case- and control-individuals, 
we used the procedure described in Bacanu et al (2000), with $\mathrm{F}_{\mathrm{st}}$ assumed to be 0.01 (Bacanu et al, 2000). The power of the family sample for the TDT analysis was modeled using PBAT software (Lange and Laird, 2002a,b). The significance level of all tests was set at $\alpha=0.05$.

\section{RESULTS}

\section{Genotypes and Alleles}

Summary statistics, by locus and sample, are given in Supplementary Table 1. The $932 \mathrm{C}>\mathrm{G}$ SNP, which was genotyped in the family dataset only, had a minor allele frequency of $2 \%$ in AN probands. This SNP was present in only 11 of the affected relatives and parents, and thus was excluded from the case:control association and transmission distortion analyses. Using duplicate samples, estimated genotyping error rates were $0.0 \%$ for all SNPs. There were no non-Mendelian transmissions at the -141 Indel or the $932 \mathrm{C}>\mathrm{G}$ SNP, one at the $939 \mathrm{C}>\mathrm{T}$ SNP and two at the 957C $>$ T SNP; the two families (total) exhibiting nonMendelian transmissions were dropped from TDT analyses. All polymorphisms genotyped in the AN proband and EAF samples were in HWE at a critical value of $P<0.05$ (data not shown). There were 25 tests of HWE conducted, though only 13 were independent tests with respect to categorical sample (the subsamples of probands with RAN and PAN diagnoses were derived from the AN proband sample).

\section{Association Analyses}

Both $+7253^{\prime} \mathrm{G}>\mathrm{T}$ and TaqIA $\mathrm{C}>\mathrm{T}$ exhibited a statistically significant genotypic association in the PAN sample $\left(\chi^{2}=5.905\right.$ and $6.683, P$-values $=0.042$ and 0.045 , respectively), although tests of allelic association were not significant (Supplementary Table 2). No other individual DRD2 SNPs exhibited statistically significant association to AN or AN subtype diagnoses. Pairwise haplotypes composed of the DRD2 -141 Indel and exon 7 SNPs $(939 \mathrm{C}>\mathrm{T}$ and $957 \mathrm{C}>\mathrm{T})$, and of the $939 \mathrm{C}>\mathrm{T}$ and $3^{\prime} \mathrm{UTR}$ SNPs $(+725$ $3^{\prime} \mathrm{G}>\mathrm{T}$ ) were significantly associated to AN diagnoses in the case:wcontrol design (Table 1). Similar haplotypic association occurs in the RAN sample with haplotypes composed of the -141 Indel and the 939C $>\mathrm{T}$ exon $7 \mathrm{SNP}$, and composed of the exon $7939 \mathrm{C}>\mathrm{T}$ and TaqIA $\mathrm{C}>\mathrm{T}$ SNPs. The frequency estimates of pairwise haplotypes composed of the 939C $>\mathrm{T}$ SNP and the two $3^{\prime}$ SNPs $\left(+7253^{\prime} \mathrm{G}>\mathrm{T}\right.$ and TaqIA $\left.\mathrm{C}>\mathrm{T}\right)$ differ between the AN proband sample and subsamples (Table 1), which may indicate haplotype frequency differences between the RAN and PAN subsamples or may be a result of the smaller sample sizes. Larger samples of individuals with RAN and PAN diagnoses, and a larger sample of control individuals would help resolve this uncertainty. Pairwise haplotype frequencies in individuals with a diagnosis of PAN and in control individuals did not exhibit statistically significant differences (Table 1).

There were 24, 68 and 82 informative families at the -141 Indel, the $939 \mathrm{C}>\mathrm{T}$, and at the $957 \mathrm{C}>\mathrm{T}$ SNP, respectively, to test for transmission distortion in the AN-ARP family dataset (Table 2). The -141 Indel and the 957C $>\mathrm{T}$ SNPs exhibited statistically significant transmission distortion (C allele over-transmitted in each case). The 939C $>$ T SNP exhibited substantial, but not significant, transmission distortion. In three-locus haplotype analysis (Table 2), four common haplotype alleles were estimated from the family data set. The most common haplotype, -141C/939C/957C (2.1.1), was significantly over-transmitted and two less common haplotypes, $-141 \mathrm{C} / 939 \mathrm{C} / 957 \mathrm{~T}$ (2.1.2) and $-141 \mathrm{Del} / 939 \mathrm{~T} / 957 \mathrm{~T}$ (1.2.2), exhibited significant undertransmission (Table 2). Permutation $P$-values were similar and the permutation $P$-value for transmission distortion of all estimated haplotypes (seven haplotypes of a possible eight haplotypes) was significant at $P=8 \times 10^{-5}$. Using the same three DRD2 polymorphisms exhibiting transmission distortion in the family-based analysis, one three-locus haplotype case : control contingency analysis was performed using the proband (case) and control samples only (data not shown); nominally significant association was observed to AN and RAN diagnosis $\left(\chi^{2}=11.309, P=0.0233\right.$, permutation $P=0.04970 \pm 0.0166$ and $\chi^{2}=8.717, P=0.0685$, permutation $P=0.04740 \pm 0.0054$, respectively).

The genotyped SNPs span a genomic region greater than $75 \mathrm{~kb}$ (Figure 1). The percentage of marker pairs with strong $D^{\prime}$-values (greater than 0.5 ) was much greater in the AN sample $(67 \%)$ than in the control sample (27\%) (Figure 2). The AN proband sample exhibits strong LD throughout the DRD2 locus, from the promoter Indel through the coding region (IVS2 and exon 7), and from the coding region through the $3^{\prime}$ region of the gene, while the control sample exhibits LD only from the coding region through the $3^{\prime}$ region of the gene. When $\mathrm{LD}$ was measured within each diagnostic subgroup, the LD patterns show some differentiation: for RAN, $53 \%$ of pairwise loci show $D^{\prime}$-values $>0.5$; for PAN, the figure is $80 \%$.

\section{DISCUSSION}

DRD2 SNPs, two of which have demonstrated in vitro functional differences, are associated with AN diagnosis in these proband, affected relative and control samples (Supplementary Table 2, Tables 1 and 2). The major allele at the -141 Indel exhibits significant linkage and association to AN diagnosis in family-based transmission disequilibrium analysis. The same allele at the -141 Indel is in excess in the AN proband sample in comparison to the unrelated control sample, although the case : control analysis is not statistically significant. The most consistent evidence for association to AN diagnosis is exhibited by multilocus SNP analyses. In these analyses, the promoter polymorphism and exon 7 SNPs (-141 Indel, 939Y and 957Y) exhibited statistically significant association in haplotype case:control analyses, differences in linkage disequilibrium estimates, and statistically significant transmission distortion. Deletion of a cytosine at -141 has been reported to reduce transcription by an average of $68 \%$ in two cell lines (Arinami et al, 1997). The 957T allele has been shown to exhibit decreased stability and reduced translation of the DRD2 transcript in vitro (Duan et al, 2003). 957T exhibits statistically significant under-transmission in the 1.2.2 haplotype in combination with the -141 deletion allele (Table 2), suggesting that the 957T allele may contribute to the association to AN diagnosis via a protective effect in this 
Table I All AN, RAN, PAN, and EAF Control Pairwise Estimated Haplotype $(H)$ Frequencies

\begin{tabular}{|c|c|c|c|c|c|c|c|c|c|c|c|c|}
\hline $\mathbf{H}$ & All AN & RAN & PAN & EAF & All AN & RAN & PAN & EAF & All AN & RAN & PAN & EAF \\
\hline & \multicolumn{3}{|c|}{ Indel and IVS2-273T>C } & \multicolumn{5}{|c|}{$939 \mathrm{C}>\mathrm{T}$ and $957 \mathrm{C}>\mathrm{T}$, exon 7} & \multicolumn{4}{|c|}{$939 \mathrm{C}>\mathrm{T}$, exon 7 and 725 bp $3^{\prime} \mathrm{G}>\mathrm{T}^{*} * * * *$} \\
\hline | 1 & 0.006 & & 0.014 & 0.026 & 0.601 & 0.649 & 0.548 & 0.589 & 0.589 & 0.683 & 0.518 & 0.591 \\
\hline 12 & 0.630 & 0.695 & 0.559 & 0.602 & 0.146 & 0.112 & 0.185 & 0.173 & 0.137 & 0.075 & 0.173 & 0.159 \\
\hline 21 & 0.047 & 0.034 & 0.059 & 0.038 & & & & & 0.274 & 0.213 & 0.309 & 0.222 \\
\hline \multirow[t]{2}{*}{22} & 0.317 & 0.271 & 0.369 & 0.334 & 0.253 & 0.239 & 0.0268 & 0.028 & & 0.028 & & 0.028 \\
\hline & \multicolumn{3}{|c|}{ Indel and 939C > T, exon 7* } & \multicolumn{5}{|c|}{ IVS2-2730T >C and 939C > T, exon 7} & \multicolumn{4}{|c|}{ 939C > T, exon 7 and TaqlA******** } \\
\hline | | & 0.004 & & 0.008 & 0.038 & 0.580 & 0.655 & 0.500 & 0.576 & 0.186 & 0.138 & 0.203 & 0.229 \\
\hline 12 & 0.745 & 0.763 & 0.724 & 0.723 & 0.058 & 0.043 & 0.073 & 0.044 & 0.550 & 0.612 & 0.517 & 0.536 \\
\hline 21 & 0.047 & 0.047 & 0.046 & 0.039 & 0.142 & 0.103 & 0.182 & 0.184 & & 0.031 & & \\
\hline 22 & 0.205 & 0.189 & 0.222 & 0.199 & 0.221 & 0.198 & 0.246 & 0.196 & 0.264 & 0.219 & 0.280 & 0.235 \\
\hline 21 & 0.050 & 0.047 & 0.04 & 0.050 & & & & 0.007 & 0.274 & 0.241 & 0.309 & 0.237 \\
\hline \multirow[t]{2}{*}{22} & 0.349 & 0.305 & 0.399 & 0.360 & 0.367 & 0.310 & 0.427 & 0.368 & 0.142 & 0.112 & 0.173 & 0.186 \\
\hline & \multicolumn{3}{|c|}{ Indel and 725 bp $3^{\prime} \mathrm{G}>\mathrm{T}$} & & \multicolumn{4}{|c|}{ IVS2-2730T > C and 725 bp 3' G>T } & \multicolumn{4}{|c|}{ 957C > T, exon 7 and TaqlA } \\
\hline | 1 & 0.042 & 0.019 & 0.070 & 0.045 & 0.634 & 0.698 & 0.565 & 0.610 & 0.041 & 0.048 & 0.034 & 0.072 \\
\hline 12 & 0.818 & $0.87 \mid$ & 0.757 & 0.765 & & & & & 0.550 & 0.589 & 0.508 & 0.512 \\
\hline 21 & 0.011 & 0.015 & 0.002 & 0.030 & 0.228 & 0.190 & 0.269 & 0.213 & 0.149 & 0.129 & 0.169 & 0.163 \\
\hline 22 & 0.129 & 0.095 & 0.170 & 0.159 & 0.138 & 0.112 & 0.167 & 0.177 & 0.260 & 0.234 & 0.288 & 0.253 \\
\hline \multicolumn{5}{|c|}{ Indel and TaqIA } & \multicolumn{3}{|c|}{ IVS2-2730T >C and TaqIA } & & \multicolumn{3}{|c|}{725 bp $3^{\prime} \mathrm{G}>\mathrm{T}$ and TaqlA } & \\
\hline
\end{tabular}

*AN vs EAF, $\chi^{2}=10.79, P=0.013$, permutation $P=0.03$; RAN vs EAF, $\chi^{2}=7.80, P=0.050$, perm. $P=0.036$; **AN vs EAF, $\chi^{2}=11.06, P=0.011$, perm. $P=0.016$; ****AN vs $E A F, \chi^{2}=8.45, P=0.038$, perm. $P=0.033$, Fisher $P=0.037$; *****AN vs EAF, $\chi^{2}=9.34, P=0.025$, perm. $P=0.021$, Fisher $P=0.023$.

sample. Thus, these data suggest that normative DRD2 transcriptional efficiency, transcript stability, and translational efficiency are associated with a diagnosis of $\mathrm{AN}$ through the joint functional effects of the DRD2 -141C (Arinami et al, 1997) and the 957C (Duan et al, 2003) alleles. Also, the $932 \mathrm{G}$ or Cys311 allele, which has been shown to exhibit significantly reduced affinity for dopamine and inhibitory response to DRD2 agonists (Cravchik et al, 1996), is associated with the $-141 \mathrm{Del}$, 939T, and 957T haplotype allele in this sample (estimated frequency 1\%), suggesting that it may contribute, in a minor fashion, as the -141 Del, 939T, and 957T haplotype is under-transmitted to individuals with a diagnosis of AN (Table 2).

Our analyses show that a sample having similar properties to the samples analyzed herein, in terms of sample size and other attributes, would have at least $80 \%$ power to detect a genetic variant accounting for $1 \%$ or more the variance of affection status. These calculations assume the frequency of the minor allele for this variant exceeds $7 \%$, Type I error is controlled at 5\%, and holds for most simple inheritance models. The exception is a recessive model, which requires a minor allele frequency more than 0.25 to achieve good power. TDT tests exhibit very similar features for power $(80 \%$ is achieved for minor allele frequency $>10 \%$, except for the recessive inheritance model). On the other hand, our results should be considered in light of several sources of error. There is the possibility of Type I error because of the substantial number of statistical tests performed. Many of these tests, however, are highly dependent due to the substantial LD observed between markers. The lack of independence of the case sample in the case: control and TDT analyses is another concern. Finally, based on the results of published linkage analyses (Grice et al, 2002; Devlin et al, 2002) of these samples, DRD2 (at $11 \mathrm{q} 22)$ does not lie in a compelling linkage region. All of these concerns underscore the necessity of confirmation from independent studies. This is also true for previous findings for HTR1D and OPRD1 on chr1p36.3-34.3 (Bergen et al, 2003a), because all of these genes have been identified as associated with AN only in our samples. Likewise the 
Table 2 DRD2 SNP and Haplotypic (- |4| Indel.939C> T.957C > T) TDT Results in RAN and PAN Probands and Affected Relatives

\begin{tabular}{|c|c|c|c|c|}
\hline \multicolumn{2}{|l|}{ SNP alleles } & $-|4|$ Indel-IC & $939 \mathrm{C}>\mathrm{T}$ & $957 \mathrm{C}>\mathrm{T}$ \\
\hline Alleles & & $1 / 2$ & $1 / 2$ & $1 / 2$ \\
\hline Observed & & $8 / 48$ & $112 / 50$ & $129 / 67$ \\
\hline Expected (S) & & $14 / 42$ & $104 / 58$ & $1 \mid 4 / 82$ \\
\hline Variance $(S)$ & & 6.917 & 24.694 & 30.713 \\
\hline$|Z|$ & & 2.472 & 1.643 & 2.737 \\
\hline$P$ & & 0.0135 & 0.1003 & 0.0062 \\
\hline DRD2 haplotype & 2.1 .1 & 2.2 .2 & 2.1 .2 & 1.2 .2 \\
\hline Estimated frequency & 0.540 & 0.209 & 0.171 & 0.069 \\
\hline$N$ informative families & 80 & 57 & 51 & 21 \\
\hline Observed & 189 & 75 & 41 & 14 \\
\hline Expected (S) & 172 & 74 & 53 & 20 \\
\hline Variance $(S)$ & 30.94 & 21.228 & 15.422 & 5.035 \\
\hline Z & 3.119 & 0.276 & -3.093 & -2.81 \\
\hline Asymptotic P (I d.f.) & 0.0018 & 0.7825 & 0.0020 & 0.0050 \\
\hline Permutation $P$ & 0.0015 & 0.8241 & 0.0020 & 0.0040 \\
\hline
\end{tabular}

Observed = number of observed transmission of alleles or haplotypes (rounded for haplotypes); expected $(S)=$ number of expected transmitted alleles or haplotypes (rounded for haplotypes); variance $(S)=$ variance of expected $(S)$; $|Z|=$ absolute value of normalized $z$ score of transmission statistic with $P=$ two sided $P$-value, I d.f; estimated frequency = frequency of the haplotype estimated by Expectation Maximization; $N$ informative families = number of families with a heterozygote transmitting (rounded); asymptotic $P=$ asymptotic $\chi^{2} P$-value; permutation $P=$ empirical $P$-value based on 6663 Monte Carlo samples.

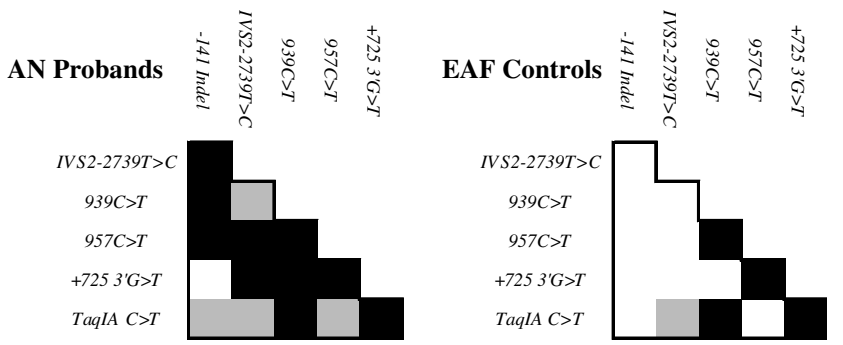

Figure 2 Point estimates of $D^{\prime}$ of $A N$ probands and EAF controls at DRD2 ( $\mathbf{\square}=0.8-1.0, \square=0.5-0.8, \square=0.0-0.5)$.

attribution of cumulative relative risk for illness for any of these genes would be premature until sufficient confirmatory evidence has been amassed by analysis of other, independent samples.

The statistically significant association between DRD2 SNPs and AN diagnosis could be taken as evidence that the D2 receptor gene is a susceptibility factor in the development of AN. Disentangling the effect of these DRD2 polymorphisms will require further association analyses in larger samples and/or functional analyses of haplotypes with more biologically based phenotypes than DSM-IV based diagnoses. For example, whether DRD2 haplotypes with these functional SNPs correlate with the clinical features of dopamine dysregulation in $\mathrm{AN}$, such as elevated anhedonia (Davis and Woodside, 2002), a diminished capacity to experience pleasure or reward (Salamone, 1996), remains an open question. In fact, $\left[{ }^{11} \mathrm{C}\right]$ raclopride neuroimaging studies show that individuals who are recovered from AN have findings consistent with elevated D2 or D3 receptor activity (Frank et al, in press). A neuroimaging study has identified a negative correlation between striatal D2 receptor availability and BMI (Wang et al, 2001). Future studies of $\left[{ }^{11} \mathrm{C}\right]$ raclopride neuroimaging studies of individuals stratified by DRD2 genotypes may help elucidate the effects of these SNPs on D2 receptor availability. We would expect to find altered expression of striatal D2 receptors in individuals with AN, in part mediated by the DRD2 -141 Indel, 957Y, and perhaps other DRD2 SNPs. That is, individuals with a diagnosis of AN and with the haplotypes identified in this sample as significantly over-, or under, transmitted should exhibit altered D2 receptor availability in $\left[{ }^{11} \mathrm{C}\right]$ raclopride imaging studies, compared to individuals without a diagnosis of $\mathrm{AN}$ and without the haplotypes identified as significantly over- or under-transmitted. Such functional analyses will contribute to a better understanding of the role of dopaminergic function in AN.

\section{ACKNOWLEDGEMENTS}

We are indebted to the participating families for their contribution of time and effort in support of this study. We wish to thank the Price Foundation for the support of the clinical collection of subjects and genotyping, and contribution to the support of data analysis. We wish to thank Neil Caporaso, MD, for comments on an earlier draft of the manuscript. Data analysis was also supported by a grant from the National Institute of Health (MH57881; BD) and a NARSAD Young Investigator Award (S-AB). Some of the results of this paper were obtained using the S.A.G.E. package of genetic epidemiology software, which is supported by a US Public Health Service Resource Grant (RR03655) from the National Center for Research Resources.

\section{REFERENCES}

Arinami T, Gao M, Hamaguchi H, Toru M (1997). A functional polymorphism in the promoter region of the dopamine D2 receptor gene is associated with schizophrenia. Hum Mol Genet 6: $577-582$.

Bacanu SA, Devlin B, Roeder K (2000). The power of genomic control. Am J Hum Genet 66: 1933-1944.

Bardo MT, Donohew RL, Harrington NG (1996). Psychobiology of novelty seeking and drug seeking behavior. Behav Brain Res 77: 23-43.

Bergen AW, van den Bree MB, Yeager M, Welch R, Ganjei JK, Haque $\mathrm{K}$ et al (2003a). Candidate genes for anorexia nervosa in the 1p33-36 linkage region: serotonin $1 \mathrm{D}$ and delta opioid receptor loci exhibit significant association to anorexia nervosa. Mol Psychiatry 8: 397-406.

Bergen AW, Yeager M, Welch R, Ganjei JK, Deep-Soboslay A, Haque $\mathrm{K}$ et al (2003b). Candidate gene analysis of the Price 
Foundation anorexia nervosa affected relative pair dataset. Curr Drug Target CNS Neurol Disord 2: 41-51.

Beumont PJ, Arthur B, Russell JD, Touyz SW (1994). Excessive physical activity in dieting disorder patients: proposals for a supervised exercise program. Int J Eat Disord 15: 21-36.

Blum K, Sheridan PJ, Wood RC, Braverman ER, Chen TJ, Comings DE (1995). Dopamine D2 receptor gene variants: association and linkage studies in impulsive-addictive-compulsive behaviour. Pharmacogenetics 5: 121-141.

Bulik CM, Tozzi F, Anderson C, Mazzeo S, Aggen S, Sullivan PF (2003). The relation between components of perfectionism and eating disorders. Am J Psychiatry 160: 366-368.

Cargill M, Altshuler D, Ireland J, Sklar P, Ardlie K, Patil N et al (1999). Characterization of single-nucleotide polymorphisms in coding regions of human genes. Nat Genet 22: 231-238.

Comings DE, Blum K (2000). Reward deficiency syndrome: genetic aspects of behavioral disorders. Prog Brain Res 126: 325-341.

Cravchik A, Goldman D (2000). Neurochemical individuality: genetic diversity among human dopamine and serotonin receptors and transporters. Arch Gen Psychiatry 57: 1105-1114.

Cravchik A, Sibley DR, Gejman PV (1996). Functional analysis of the human D2 dopamine receptor missense variants. J Biol Chem 271: 26013-26017.

Davis C, Woodside DB (2002). Sensitivity to the rewarding effects of food and exercise in the eating disorders. Comp Psychiatry 43: 189-194.

Devlin B, Bacanu SA, Klump KL, Bulik CM, Fichter MM, Halmi KA et al (2002). Linkage analysis of anorexia nervosa incorporating behavioral covariates. Hum Mol Genet 11: 689-696.

Duan J, Wainwright MS, Comeron JM, Saitou N, Sanders AR, Gelernter J et al (2003). Synonymous mutations in the human dopamine receptor D2 (DRD2) affect mRNA stability and synthesis of the receptor. Hum Mol Genet 12: 205-216.

Elston RC (2001). S.A.G.E. Statistical Analysis for Genetic Epidemiology. MetroHealth Campus, Case Western Reserve University: Cleveland.

Excoffier L, Slatkin M (1995). Maximum-likelihood estimation of molecular haplotype frequencies in a diploid population. $\mathrm{Mol}$ Biol Evol 12: 921-927.

Fichter MM, Herpertz S, Quadflieg N, Herpertz-Dahlmann B (1998). Structured interview for anorexic and bulimic disorders for DSM-IV and ICD-10: updated (third) revision. Int J Eat Disord 24: 227-249.

First MB, Gibbon M, Spitzer RL, Williams JB (1996). User's guide for the structured clinical interview for DSM IV axis I disordersresearch version SCID-I, version 2.0. Biometrics Research, New York Stat Psychiatric Institute: New York.

Frank GK, Bailer UF, Henry SE, Drevets W, Meltzer CC, Price JC et al (in press). Increased Dopamine D2/D3 receptor binding after recovery from Anorexia Nervosa measured by positron emission tomography and $\left[{ }^{11} \mathrm{C}\right]$ raclopride. Biol Psychiatry.

Fujiwara Y, Yamaguchi K, Tanaka Y, Tomita H, Shiro Y, Kashihara $\mathrm{K}$ et al (1997). Polymorphism of dopamine receptors and transporter genes in neuropsychiatric diseases. Eur Neurol 38(Suppl 1): 6-10.

Grandy DK, Litt M, Allen L, Bunzow JR, Marchionni M, Makam H et al (1989). The human dopamine $\mathrm{D} 2$ receptor gene is located on chromosome 11 at q22-q23 and identifies a TaqI RFLP. Am J Hum Genet 45: 778-785.

Grice DE, Halmi KA, Fichter MM, Strober M, Woodside DB, Treasure JT et al (2002). Evidence for a susceptibility gene for anorexia nervosa on chromosome 1. Am J Hum Genet 70: 787-792.

Halmi KA, Sunday SR, Strober M, Kaplan A, Woodside DB, Fichter $M$ et al (2000). Perfectionism in anorexia nervosa: variation by clinical subtype, obsessionality, and pathological eating behavior. Am J Psychiatry 157: 1799-1805.
Itokawa $\mathrm{M}$, Arinami T, Futamura N, Hamaguchi $\mathrm{H}$, Toru $\mathrm{M}$ (1993). A structural polymorphism of human dopamine D2 receptor, D2(Ser311 $\rightarrow$ Cys). Biochem Biophys Res Commun 196: 1369-1375.

Kalivas PW (1993). Neurotransmitter regulation of dopamine neurons in the ventral tegmental area. Brain Res Brain Res Rev 18: 75-113.

Kaye WH, Bulik CM, Thornton L, Barbarich N, Masters K (2004). Comorbidity of anxiety disorders with anorexia and bulimia nervosa. Am J Psychiatry 161: 2215-2221.

Kaye WH, Frank GK, McConaha C (1999). Altered dopamine activity after recovery from restricting-type anorexia nervosa. Neuropsychopharmacology 21: 503-506.

Kaye WH, Lilenfeld LR, Berrettini WH, Strober M, Devlin B, Klump KL et al (2000). A search for susceptibility loci for anorexia nervosa: methods and sample description. Biol Psychiatry 47: 794-803.

Kebabian JW, Calne DB (1979). Multiple receptors for dopamine. Nature 277: 93-96.

Khan ZU, Mrzljak L, Gutierrez A, de la Calle A, Goldman-Rakic PS (1998). Prominence of the dopamine D2 short isoform in dopaminergic pathways. Proc Natl Acad Sci USA 95: 7731-7736.

Kidd KK, Morar B, Castiglione CM, Zhao H, Pakstis AJ, Speed WC et al (1998). A global survey of haplotype frequencies and linkage disequilibrium at the DRD2 locus. Hum Genet 103: 211-227.

Klump KL, Miller KB, Keel PK, McGue M, Iacono WG (2001). Genetic and environmental influences on anorexia nervosa syndromes in a population-based twin sample. Psychol Med 31: 737-740.

Kortegaard LS, Hoerder K, Joergensen J, Gillberg C, Kyvik KO (2001). A preliminary population-based twin study of selfreported eating disorder. Psychol Med 31: 361-365.

Kron L, Katz JL, Gorzynski G, Weiner H (1978). Hyperactivity in anorexia nervosa: a fundamental clinical feature. Comp Psychiatry 19: 433-440.

Lange C, Laird NM (2002a). On a general class of conditional tests for family-based association studies in genetics: the asymptotic distribution, the conditional power, and optimality considerations. Genet Epidemiol 23: 165-180.

Lange C, Laird NM (2002b). Power calculations for a general class of family-based association tests: dichotomous traits. Am J Hum Genet 71: 575-584.

Lilenfeld LR, Kaye WH, Greeno CG, Merikangas KR, Plotnicov K, Pollice C et al (1998). A controlled family study of anorexia nervosa and bulimia nervosa: psychiatric disorders in firstdegree relatives and effects of proband comorbidity. Arch Gen Psychiatry 55: 603-610.

Morin PA, Saiz R, Monjazeb A (1999). High-throughput single nucleotide polymorphism genotyping by fluorescent $5^{\prime}$ exonuclease assay. Biotechniques 27: 538-540, 542, 544 passim.

Mukherjee J, Yang ZY, Brown T, Roemer J, Cooper M (1996). 18F-desmethoxyfallypride: a fluorine-18 labeled radiotracer with properties similar to carbon-11 raclopride for PET imaging studies of dopamine D2 receptors. Life Sci 59: 669-678.

O'Connell JR, Weeks DE (1998). PedCheck: a program for identification of genotype incompatibilities in linkage analysis. Am J Hum Genet 63: 259-266.

Rousset F, Raymond M (1995). Testing heterozygote excess and deficiency. Genetics 140: 1413-1419.

Salamone JD (1996). The behavioral neurochemistry of motivation: methodological and conceptual issues in studies of the dynamic activity of nucleus accumbens dopamine. J Neurosci Methods 64: 137-149.

Sarkar G, Kapelner S, Grandy DK, Marchionni M, Civelli O, Sobell J et al (1991). Direct sequencing of the dopamine D2 
receptor (DRD2) in schizophrenics reveals three polymorphisms but no structural change in the receptor. Genomics 11: 8-14.

Schneider S, Roessli D, Excoffier L (2000). Arlequin Ver. 2.000: A Software for Population Genetic Analysis. Genetics and Biometry Laboratory, University of Geneva: Switzerland. http://anthro. unige.ch/alrequin.

Sohlberg S, Strober M (1994). Personality in Anorexia nervosa: an update and a theoretical integration. Acta Psychiatr Scand Suppl 378: $1-15$.

Strober M, Freeman R, Lampert C, Diamond J, Kaye W (2000). Controlled family study of anorexia nervosa and bulimia nervosa: evidence of shared liability and transmission of partial syndromes. Am J Psychiatry 157: 393-401.

Sullivan PF (1995). Mortality in anorexia nervosa. Am J Psychiatry 152: $1073-1074$.
Szczypka MS, Rainey MA, Kim DS, Alaynick WA, Marck BT, Matsumoto AM. et al (1999). Feeding behavior in dopaminedeficient mice. Proc Natl Acad Sci USA 96: 12138-12143.

Szczypka MS, Rainey MA, Palmiter RD (2000). Dopamine is required for hyperphagia in Lep(ob/ob) mice. Nat Genet 25: 102-104.

Usiello A, Baik JH, Rouge-Pont F, Picetti R, Dierich A, LeMeur M et al (2000). Distinct functions of the two isoforms of dopamine D2 receptors. Nature 408: 199-203.

Wade TD, Bulik CM, Neale M, Kendler KS (2000). Anorexia nervosa and major depression: shared genetic and environmental risk factors. Am J Psychiatry 157: 469-471.

Wang GJ, Volkow ND, Logan J, Pappas NR, Wong CT, Zhu W et al (2001). Brain dopamine and obesity. Lancet 357: 354-357.

Wise RA, Bozarth MA (1984). Brain reward circuitry: four circuit elements 'wired' in apparent series. Brain Res Bull 12: 203-208.

Supplementary Information accompanies the paper on the Neuropsychopharmacology website (http://www.nature.com/ npp). 